\title{
An Open Framework for Extensible Multi-stage Bioinformatics Software
}

\author{
Gabriel Keeble-Gagnère ${ }^{1}$, Johan Nyström-Persson ${ }^{2}$, \\ Matthew I. Bellgard ${ }^{1}$, and Kenji Mizuguchi ${ }^{2}$ \\ ${ }^{1}$ Centre for Comparative Genomics, Murdoch University, Australia \\ 2 National Institute of Biomedical Innovation, Japan
}

\begin{abstract}
In research labs, there is often a need to customise software at every step in a given bioinformatics workflow, but traditionally it has been difficult to obtain both a high degree of customisability and good performance. Performance-sensitive tools are often highly monolithic, which can make research difficult. We present a novel set of software development principles and a bioinformatics framework, Friedrich, which is currently in early development. Friedrich applications support both early stage experimentation and late stage batch processing, since they simultaneously allow for good performance and a high degree of flexibility and customisability. These benefits are obtained in large part by basing Friedrich on the multiparadigm programming language Scala. We present a case study in the form of a basic genome assembler and its extension with new functionality. Our architecture 1 has the potential to greatly increase the overall productivity of software developers and researchers in bioinformatics.
\end{abstract}

\section{Introduction}

Bioinformatics poses a particularly difficult challenge for software developers, with constantly changing end-user requirements and the need to interact with an ever-expanding range of tools and data formats. The advent of big data means that the tools and skills required for data manipulation and basic research are now more advanced than before. However, researchers are fundamentally biologists and more interested in the data itself than in addressing technical issues, which traditionally fall into the computer science field. The challenge for software developers is thus to put the maximum amount of power and flexibility in the hands of the users while assuming as little technical knowledge as possible.

When large data volumes are processed, high performance software tools are often used. However, such tools are often highly specialised and optimised for a specific purpose, permitting only limited customisation. This kind of software is often also monolithic. Monolithic tools can be efficient for handling big data problems, but such a design often runs counter to a natural research process,

${ }^{1}$ Available freely under a dual GPL/MIT open-source license from https://bitbucket.org/jtnystrom/friedrich/. 
since researchers often need to make adjustments to various parts of the tools that they work with, particularly in fast-changing fields such as bioinformatics. MacLean and Kamoun [8], reporting on their experience bringing a small bioinformatics laboratory into the age of big data, state that biologists at first tend to regard bioinformatics processes as being monolithic, but once they understand their inner workings generally become more productive, especially if they can take charge of tools and methods themselves to some degree. Clearly, transparent and flexible tools have the potential to play a very important role.

We argue that it is possible to develop software that makes researchers more productive and enables them to ask more questions about their data and their process by adopting a new set of software development principles. In the following, we present the Friedrich architecture (Section 2). We then discuss the Friedrich framework, a toolkit for building bioinformatics applications according to these principles (Section 3). We discuss the implementation of a basic genome assembler based on Friedrich in Section 4. We compare with other tools and frameworks in Section 5 and conclude the paper in Section 6 .

\section{The Friedrich Software Principles}

The Friedrich architecture is a set of interlocking software design principles that, in our view, can support bioinformatics research very effectively.

Expose Internal Structure. Bioinformatics software should expose its internal building blocks and data flow to a high degree, permitting reconfigurability. Bioinformatics computation often consists of sending data through a number of processing stages until the desired output is produced. Frameworks should reflect this by consisting of modules that can easily be rewired - reconnected in different sequences - to represent changing workflows. This is the opposite of a monolithic application, which is effectively a black box.

Conserve Dimensionality Maximally. The processing of a given data set which can essentially be viewed as a set of points in a mathematical space - to produce a given output, is analogous to a projection in geometry. For example, in $\mathbb{R}^{3}$, the equation

$$
x_{1}^{2}+x_{2}^{2}+x_{3}^{2}=1
$$

defines a sphere of radius 1 centred at the origin. The projection $\operatorname{proj}_{1}$, which sends $\left(x_{1}, x_{2}, x_{3}\right) \in \mathbb{R}^{3}$ to $x_{1} \in \mathbb{R}$, when applied to the sphere defined above, yields: $x_{1}^{2}=1$, which defines the set of two points $\{-1,1\}$. If $f: \mathbb{R}^{3} \rightarrow \mathbb{R}^{3}$ is a mapping, then given a surface in $\mathbb{R}^{3}$ (such as the sphere defined above), the function $\operatorname{proj}_{1} \circ f$ returns an answer to the query "At what points does the mapped surface intersect the $x_{1}$-axis?" Given an answer to the query, we cannot extract information about the original surface. In an analogous way, raw bioinformatics data contains all possible information from a given experiment. Thus it has maximum dimensionality. As various data processing is performed on this data set, its dimensionality is reduced. For example, given a set of reads 
from a DNA sequencing run, one processing step might be to remove duplicates, to produce a set of non-redundant sequence reads. This would clearly reduce the dimensionality of the resultant data set, since the redundancy information is lost.

Maximal conservation of dimensionality permits users who are applying tools experimentally to go back to previous stages of their computation and attempt different parameters, adding a great deal of flexibility to the experimental process, allowing new questions to be asked, and saving time. It can also be thought of as maximal preservation of the results of intermediate phases in the computation.

Multi-stage Applications. Many tools need to be used in at least two different stages, which may loosely be called experimentation and production. In the experimental stage, researchers explore newly available data in order to develop methods and a basic understanding of what can be done. It is in this stage that the need for customisation and flexibility is greatest. In the production stage, a repeatable process is extracted and applied systematically a large number of times. In this stage there is less need for flexibility; instead, robustness, reliability, and performance are valued. However, a given analysis or tool, once developed, often has to move across this boundary from the experimental stage to the production stage. This transition is often nontrivial given that hitherto, incompatible technologies have often been used in the two stages. In such a situation, one may opt to use experimental stage technologies in both stages, resulting in poor performance. Alternatively, one may use production-stage technologies in both stages, resulting in difficulty of experimentation. Finally, one may re-develop the analysis from scratch once it makes the transition, which would be a large additional effort.

Friedrich software should support a full range of development stages, including experimentation, production, and any intermediate points. Because a single technology framework is used consistently, it becomes easy to move from experimentation to production, and also to move back again. This enables a feedback loop between experimental usage and production usage: when something unexpected occurs in the large scale application of a tool, it can easily be taken back to the workbench for inspection, and any adjustments made can be propagated back again. Table 1 gives a comparison.

Table 1. A comparison of Friedrich's target characteristics with tools designed mainly for either experimentation or production

\begin{tabular}{|l|l|l|l|l|}
\hline Context & $\begin{array}{l}\text { Necessary } \\
\text { flexibility }\end{array}$ & $\begin{array}{l}\text { Typical program- } \\
\text { ming language }\end{array}$ & Performance & Examples \\
\hline $\begin{array}{l}\text { Experimental } \\
\text { stage tools }\end{array}$ & High & Perl, Python, R, & Low/ moderate & $\begin{array}{l}\text { BioPerl, } \\
\text { BioPython }\end{array}$ \\
\hline $\begin{array}{l}\text { Production stage } \\
\text { tools }\end{array}$ & Low & C, C++, Java, ... & Very high & $\begin{array}{l}\text { Velvet, Abyss, } \\
\text { BioJava }\end{array}$ \\
\hline Friedrich & High & Scala, Java & High & Section 4 \\
\hline
\end{tabular}


Flexibility with Performance. This is closely related to the previous principle. If programming languages have traditionally been separable into on one hand a category of high-performing but inflexible ones (in that applications written in them are relatively hard to customise) and on the other a category of poorly performing but flexible ones, we believe that the relatively recent language Scala (see Section 3.1) is an outlier that provides for both good performance and high flexibility. This enables flexibility with performance. For many bioinformatics applications, one should not seek extreme performance or extreme flexibility but good levels of both.

Minimal Finality. Monolithic software often makes unsustainable assumptions about data formats, algorithm parameters and data sizes. For example, the socalled next generation of sequencing equipment is expected to render many of the current genome analysis software tools unusuable, largely for the reason that certain quantity and size parameters will change. Friedrich applications should assume a minimum of finality. Software developers should not dictate how the framework or its building blocks should ultimately be used, since they cannot possibly anticipate all the usage scenarios that may eventually appear. MacLean and Kamoun found that reorienting research from a top-down model to a bottom-up model helped increase productivity in the Sainsbury Laboratory [8]. Minimising finality also helps achieve this end.

Ease of Use. Friedrich applications should not be hard for novices to use. They should provide sensible defaults at all times, so that new users can deploy them in common use cases with little effort. Simplicity should not be sacrificed to the other principles.

We have now described the software design principles of the Friedrich architecture. Next, we describe our implementation of the Friedrich framework, as well as an application built on top of it.

\section{The Friedrich Framework}

The Friedrich framework is implemented in the form of a Scala library that permits users to develop bioinformatics applications easily. In implementing this framework, our aim has been to allow application developers to follow the principles we outlined in the previous section easily. The framework is still under development, and this section describes its current state.

\subsection{The Scala Programming Language}

An early decision was made to base Friedrich on Scala, a novel programming language for the Java virtual machine, which is being developed by Martin Odersky and others [12] (http://www.scala-lang.org). Programming languages are traditionally classified as functional or imperative. Functional languages emphasise avoidance of side effects and composition of functions. Imperative languages, such as Perl, C, and Java, have been more widely used in the mainstream, 
and generally functions in these languages may have side effects. Scala blends these two paradigms. It provides libraries, constructs and idioms for stateless, purely functional programming as well as for stateful, imperative, object-oriented programming. Scala code is often very compact compared with equivalent Java code, and, provided that the programmer is somewhat disciplined, can be highly readable.

Scala brings several important benefits to Friedrich.

- Scala provides for high programmer productivity and is very well suited to big data tasks, performing well [5] even under heavy loads, thanks to the maturity of the underlying Java platform.

- Existing Java libraries for tasks such as graph processing, database access, calculation and so on can be taken advantage of immediately.

- Because of its strong support for functional programming and immutable state, Scala is a foundation that lends itself well to parallel processing, the need for which cannot be ignored in bioinformatics today.

Scala has much of the flexibility and productivity of scripting languages such as Ruby, Python and Perl. For example, Scala has features such as an interactive interpreter with auto-completion, pattern matching and convenient regular expression support. Type inference means that types in many cases do not need to be declared. SBT (Simple Build Tool), which is widely used by the Scala community, permits automatic dependency management and library downloading in a style that resembles Perl's well-known CPAN package repository.

In a survey of software engineering techniques used in 22 different bioinformatics software projects, Rother et al. described 12 practices that were found to be useful [14]. Scala and Friedrich directly support many of these, benefiting both from the mature development tools available for the Java platform and from its own tools. For example, Scala has good support for unit testing and a sophisticated documentation generator, and Friedrich supports practices such as frequent release and feedback cycles, since it enables easy transitions between the experimental and production stages.

\subsection{Friedrich Application Components}

Friedrich contains the following key components for building applications.

Phases and Pipelines. Friedrich applications are organised as sets of phases, according to the model illustrated in Figure1. Sequences of phases are called pipelines. Friedrich provides foundational classes that can be extended to implement new phases, as well as functions for managing and running pipelines.

Data Object Classes. Friedrich phases operate on standardised data objects. For a given application, all experimental data as well as configuration parameters is stored in these objects.

Configuration Management. Pipelines and general application parameters are stored in XML configuration files (Figure 1). Friedrich provides facilities for reading these configurations and automatically creating pipelines from them. 
Core Bioinformatics Functionality. Friedrich provides a small library of core bioinformatics algorithms and data representations.

In order to implement a new Friedrich application, one should select a data object type or define a new one, implement the necessary phases, and write a main method that invokes a pipeline using the Friedrich API. As we will see, implementing phases is not difficult.

Phases receive input that they make certain assumptions about (phase preconditions), perform some computation on it, and then pass on this data in a new state (phase postconditions) as output. For example, our genome assembler makes use of phases such as ScanReads, BuildGraph and FindPaths, among others (shown in Figure 2). Phases can perform almost any functionality. In accordance with our dimensionality principle, phases should add information to the shared data object rather than remove or overwrite. This permits the user to explore and manipulate the data (in interactive mode) in between pipeline phases. Friedrich applications can easily invoke pipelines based on their names only, which means that workflows can be changed without recompiling an application.

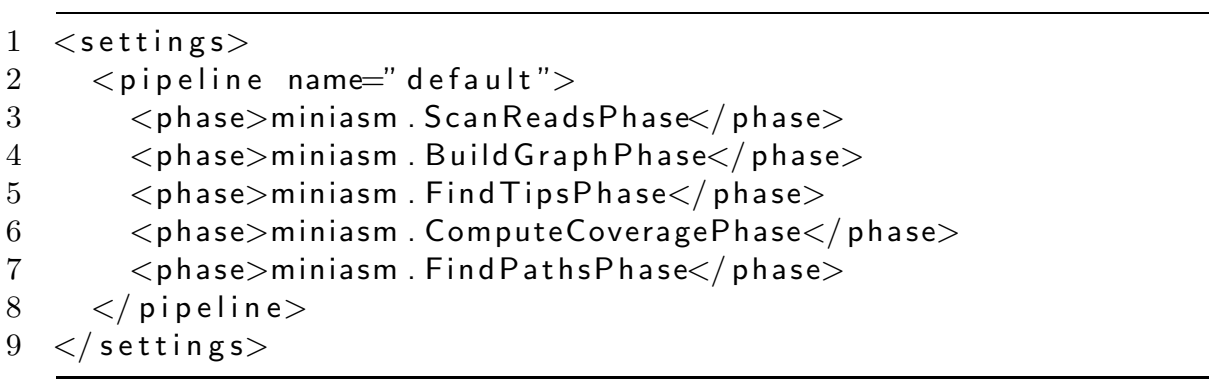

Fig. 1. An example of a pipeline configuration. The phases will be run in the order shown. 'Miniasm' is the package name of the corresponding classes.

The components we have described support the six principles as much as possible. Phases and pipelines are a natural way to expose structure. When an application is made up of a set of relatively independent phases, it becomes clear what its internal parts are, and the configuration system permits them to be rewired easily. Conservation of dimensionality is not enforced by the framework itself. Phase implementors are recommended to always add data to the shared data object and not overwrite or remove it unless necessary. In the future, we plan to provide automatic data management facilities to assist interactive use. Multi-stage applications and flexibility with performance are benefits that we derive largely from our use of the Scala language, as outlined above. Minimal finality is something we obtain in part from Scala, and in part from the pipeline and phase system, since the overall data flow of an application can be changed at a late stage. Ease of use is a principle to be upheld by application developers. 


\section{Genome Assembly with Friedrich}

Genome assembly refers to the process of turning raw sequence reads - produced from a sequencing run - into contiguous regions of DNA, know as contigs, that represent the original genome being analysed. In particular, de novo genome assembly refers to assembling a novel genome for the first time directly from individual reads - that is, without a reference genome to guide it. Assembly methods have evolved from the overlap-layout-consensus (OLC) method (employed by early sequencing efforts, including the Human Genome Project, which took advantage of the long reads produced by traditional Sanger technology) to the de Bruijn graph methods employed by most assemblers that accept current high-throughput short read data. For the technical details of genome assembly, we refer the reader to [2]. In short, the nodes of the de Bruijn graph are sequences of length $k$ base pairs (known as $k$-mers); an edge exists between two $k$-mers if their sequence overlaps by $k-1$ bases. This graph is then processed and contigs read off directly as non-ambiguous paths.

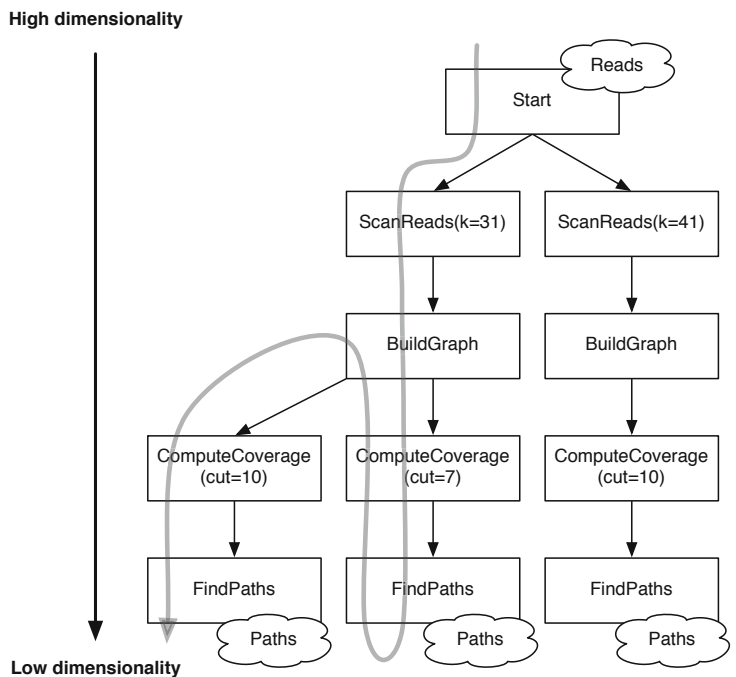

Fig. 2. The internal data flow of a hypothetical genome assembler. The grey path illustrates how a user may wish to try a number of different coverage cutoff values, which involves returning to a previous phase.

One of the early motivations for Friedrich was the desire to investigate in detail the inner workings of this process. Investigating assemblies with commonly used assemblers such as Velvet [17] and ABySS [15], we found that output can vary considerably given the same input data. As well as this, we found that outputs could vary even on very small toy data sets (data not shown). Indeed, anyone who has used these tools will be aware that different assemblers produce different 
output, but rarely will the user have a clear idea of what exactly has been done differently.

Figure 2 outlines a simplified typical workflow for an assembler. Internally, data is sent through a number of phases in order to produce the final output. As a rule, the output of each phase is less complex than its input, and the final output is much simpler than the initial input. This can be understood as a successive reduction of the dimensionality of the data. Each phase within a tool such as this assembler can be controlled by parameters (for example $k$, cut), and modifying the parameters of a phase might affect the final output significantly. Thus, researchers might want to traverse what we might call a phase tree following the curved arrow in order to compare outputs resulting from various configurations. In a monolithic tool, this is generally not possible, since one cannot return to earlier phases in the pipeline: the tool must be re-run from the starting point even when only parameters of late phases are changed, if they can be changed at all. With Friedrich, it is possible to interrogate the assembly at every step of the way.

The Friedrich-based assembler that we have developed consists of an efficient representation of sequences and reads, 11 processing phases and various utility classes. The source code is about 3000 lines in length.

\subsection{Interactive Use}

The following is an example of an interactive Friedrich session to process Illumina short read data 2 . We launch the interactive Friedrich console using SBT. If the source code of any phases or libraries being used has changed when Friedrich is launched in this way, they will automatically be recompiled, permitting a smooth development and testing workflow. The interactive Scala environment has features such as tab-completion to show all available alternatives. This environment evaluates Scala expressions as they are typed in, and allows for functions and classes to be defined on the fly.

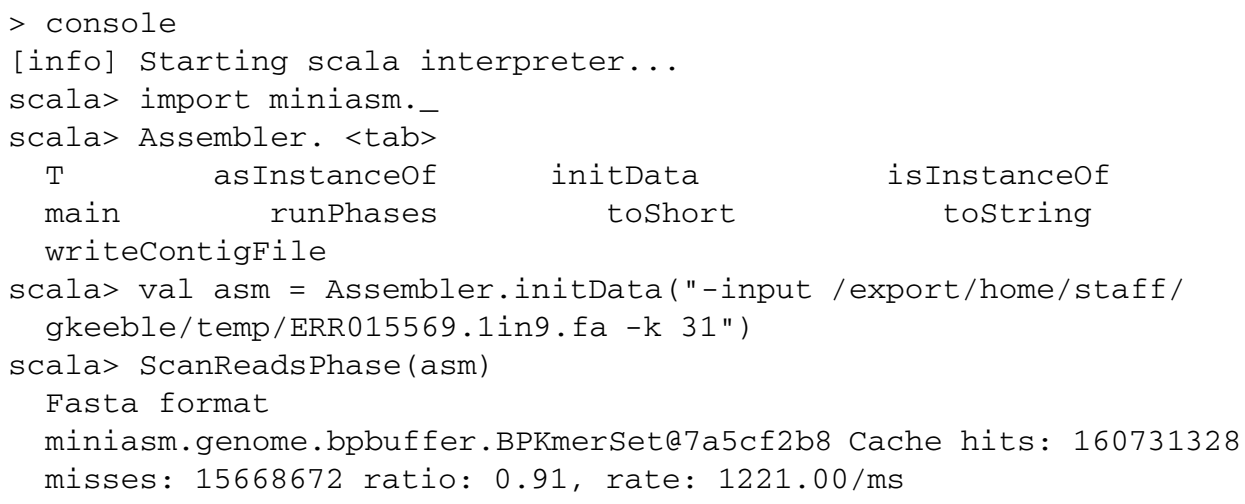

${ }^{2}$ NCBI SRA experiment ERX005938, run ERR015569. Only 1/9 of the reads were passed to Friedrich. 


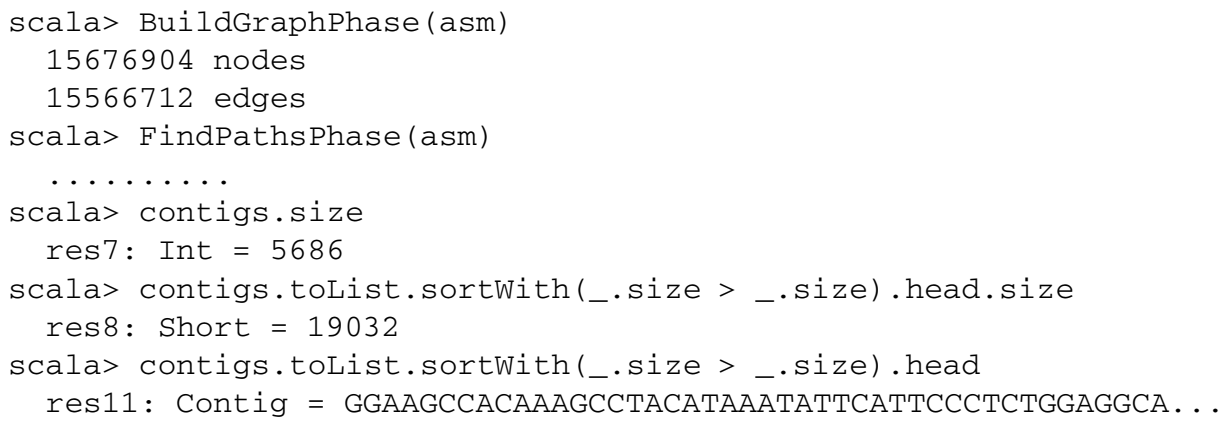

In this interactive session, we first prepare a data object using Assembler.initData. This method takes the same parameters that the Friedrich assembler accepts when run in non-interactive mode. The resulting AssemblyData object asm is then manually passed to different phases by the user. At any time, the user can construct additional data objects and compare them or interrogate them more closely. After FindPathsPhase has finished running, contiguous paths will be available in the asm data object. We can now use the full power of Scala to explore or alter the data that has become available. First we ask for the number of contigs that were found (5686). Then we sort the contigs to have the largest first, defining a sort function on the fly (_.size >_.size, called an anonymous function) and asking for its size (19032). Finally we examine the actual base pairs in this long contig.

\subsection{Extending the Assembler for Motif Recognition}

We now show how to extend the assembler with a phase that detects and displays repeating motifs in the contiguous base pair sequences (contigs) that have been found. In Scala, traits are a basic unit of composition. Classes and traits can inherit from multiple traits simultaneously. In this way, one can build up a family of traits, each one representing a functionality, and compose them as needed. Phases must extend a basic Friedrich phase trait called Phase. In our assembler, which is an application that is built on top of Friedrich, we define AsmPhase, which extends Phase and make it the convention that all our assembly phases will extend this new trait. Thus, we now add a phase called FindRepeatsPhase (Figure 3).

The method runlmpl implements the concrete functionality of each phase. Lines 7-12 show a generalised for-comprehension, a special feature of Scala. The variable c iterates over all contigs that have previously been found in the assembly. These are taken from the data object, which has previously been operated on by other phases. The notation (start, length, pattern) declares a 3-tuple of three variables. These will iterate over the repeated motifs returned by the method c.repeats for each value of c. When repeated motifs are found, they are printed to the console. This short snippet demonstrates that in many cases, Scala code can be considerably more compact than corresponding Java code (not shown). Also note the default values of the parameters: $\min$ TotLen: Int $=8$, minMotifLen: 


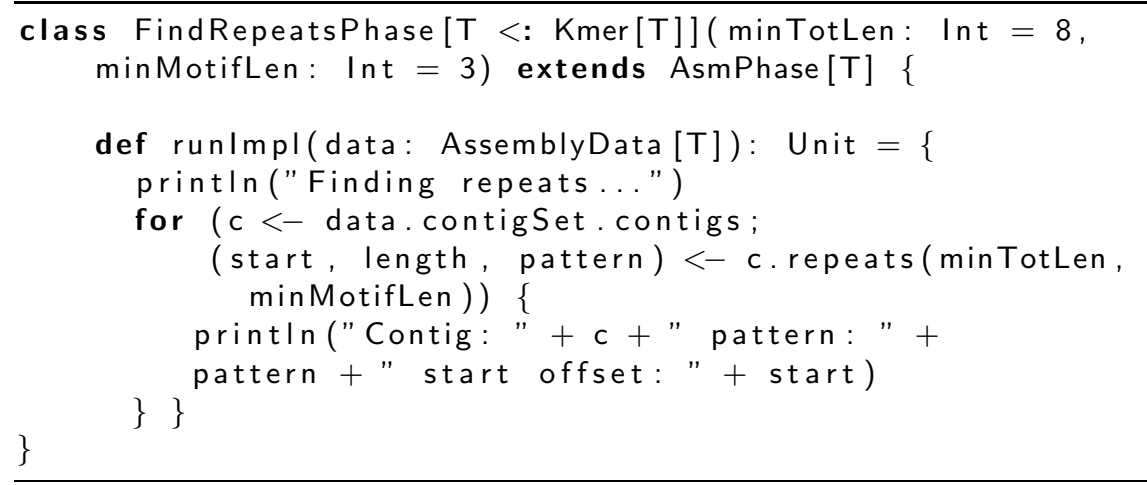

Fig. 3. The newly added FindRepeatsPhase

Int $=3$. These are the two parameters that constructors for this class take, but since they have default values, they can be omitted if needed.

Since the runlmpl method can contain any Scala code, it has access to the full range of Java and Scala APIs. Here we obtain the desired repeats by using the method c.repeats, which is defined in Friedrich's contig class (not shown). However, we are in no way limited to using only such built-in methods.

After the new phase has been defined in this fashion, no additional work is needed. It can be included in pipelines, as shown in Figure1. It can also be used interactively. We have written a small convenience function (not shown) to allow the new phase to be invoked by simply referring to its name. After assembly has been carried out, as shown in our previous interactive example (Section 4.1), we can apply the new phase:

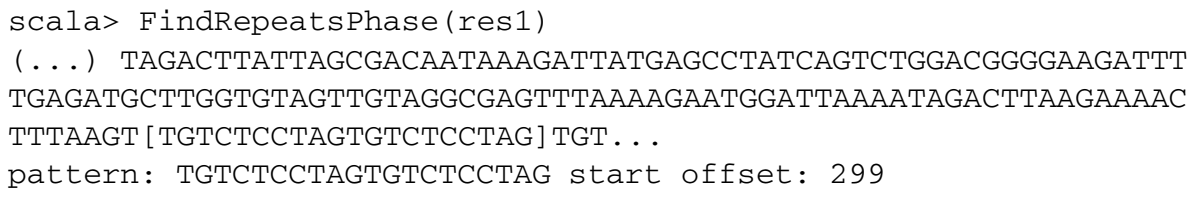

A large number of repeated motifs are found in the contigs that were previously assembled; we show one of them here. At this point, it is possible to retain the data that has been produced so far, make adjustments, and assemble again with different parameters. One can then easily contrast repeated motifs that are produced by different assembly configurations, all without leaving Friedrich.

\section{Comparison with Other Tools and Libraries}

Friedrich has similarities with many existing tools and frameworks, although we believe that there are no well-known bioinformatics tools precisely filling Friedrich's role at the moment. The pipeline and phase structure is similar to a class of software that might be called toolkits. These packages consist of individual specialised programs that operate on a shared file format. The user is free 
to run the programs in any order and can thus create their own workflow, perhaps through shell scripting. Examples of such toolkits are SAMtools/Picard [7] and GATK [10, for handling nucleotide sequence alignments. While these toolkits come close in spirit to the Friedrich design, one essential difference is that our ability to run Friedrich phases in an interactive Scala environment permits users to very easily inspect and modify data manually in between phases. Unsupported extensions to a toolkit such as SAMtools require first writing a new program from scratch, and interactive experimentation would require even more additional work. Friedrich minimises the cost of free experimentation with data as it is being processed. Note that Picard and GATK provide Java APIs, which could be easily integrated into Friedrich.

There are many general frameworks for bioinformatics, such as BioJava 4, BioPython[1], BioPerl[16, BioScala 13] and BioRuby[11. These are all utility libraries of varying size and scope, aimed at bioinformatics tasks in the respective programming language. Mangalam provides an informative comparison of the first three [9]. Although this survey is now ten years old, most of the points it makes about programming language differences are still essentially valid. However, its conclusion that BioPerl is sufficient for about $90 \%$ of bioinformatics programming needs is now outdated, with the need to process ever larger data sets. In general, the Bio-* toolkits provide useful routines and data models but do not prescribe any specific software development style. Therefore, they are somewhat orthogonal to our effort, which aims to provide both an architectural style and foundational libraries to support it. The Bio-* toolkits can in principle be integrated into Friedrich applications, in particular BioScala and BioJava. Bioinformatics workflow systems, for example those provided in Yabi [6] and Galaxy[3], are user-friendly ways of managing and applying high level computation pipelines. However, in their focus on ready-made, finalised modules they are quite different from what Friedrich seeks to become.

\section{Conclusion and Remarks}

We have argued for the introduction of a new set of software development principles for bioinformatics software, and we provide a framework that supports application development based on these principles. We have also shown an existing application based on the framework. Principles such as conservation of dimensionality and an exposed internal structure will allow developers to produce software that is more useful to bioinformaticians and better suits the research process. While Friedrich does not aim to provide either the highest performance or the greatest flexibility, with good levels of both it represents a new tradeoff that should be considered an important option for many areas in bioinformatics.

We view the architectural principles presented in Section 2 as essentially complete. However, the Friedrich software framework is still in an early stage of its development and many enhancements and extensions have yet to be implemented. For example, the BioJava [4, and BioScala 13] libraries provide a large amount of functionality for bioinformatics applications, and when doing so is 
suitable, it would be natural to "wrap" this functionality as Friedrich phases, rather than reimplement the functionality from scratch in Friedrich.

It remains to develop more applications on top of Friedrich, in addition to the genome assembler we have discussed in this work, in order to verify that the design principles hold up across a wider range of tasks in practice.

\section{References}

1. Cock, P.J.A., et al.: Biopython: freely available Python tools for computational molecular biology and bioinformatics. Bioinformatics 25(11), 1422-1423 (2009)

2. Compeau, P.E.C., et al.: How to apply de Bruijn graphs to genome assembly. Nature Biotechnology 29(11), 987-991 (2011)

3. Goecks, J., et al.: Galaxy: a comprehensive approach for supporting accessible, reproducible, and transparent computational research in the life sciences. Genome Biology 11(8), R86+ (2010)

4. Holland, R.C.G., et al.: BioJava: an Open-Source Framework for Bioinformatics. Bioinformatics 24(18), 2096-2097 (2008)

5. Hundt, R.: Loop Recognition in $\mathrm{C}++/$ Java/Go/Scala. In: Proceedings of Scala Days 2011 (2011)

6. Hunter, A.A., et al.: Yabi: An online research environment for grid, high performance and cloud computing. Source Code for Biology and Medicine 7(1), 1+ (2012)

7. Li, H., et al.: The Sequence Alignment/Map format and SAMtools. Bioinformatics 25(16), 2078-2079 (2009)

8. MacLean, D., Kamoun, S.: Big data in small places. Nature Biotechnology 30(1), 33-34 (2012)

9. Mangalam, H.: The Bio* toolkits-a brief overview. Briefings in Bioinformatics 3(3), 296-302 (2002)

10. McKenna, A., et al.: The Genome Analysis Toolkit: A MapReduce framework for analyzing next-generation DNA sequencing data. Genome Research 20(9), 12971303 (2010)

11. Mitsuteru, N.G., et al.: BioRuby: open-source bioinformatics library (2003)

12. Odersky, M.: The Scala Language Specification, Version 2.9 (May 2011), http://www.scala-lang.org/docu/files/ScalaReference.pdf

13. Prins, P.: BioScala (March 2011), https://github.com/bioscala/bioscala

14. Rother, K., et al.: A toolbox for developing bioinformatics software. Briefings in Bioinformatics 13(2), 244-257 (2012)

15. Simpson, J.T., et al.: ABySS: a parallel assembler for short read sequence data. Genome research 19(6), 1117-1123 (2009)

16. Stajich, J.E., et al.: The Bioperl toolkit: Perl modules for the life sciences. Genome research 12(10), 1611-1618 (2002)

17. Zerbino, D.R., Birney, E.: Velvet: Algorithms for de novo short read assembly using de Bruijn graphs. Genome Research 18(5), 821-829 (2008) 